\title{
Window-of-Opportunity Trials: The Road Forward in Soft Tissue Sarcoma and Beyond
}

\author{
Emily Z. Keung, MD and Christina L. Roland, MD, MS \\ Department of Surgical Oncology, The University of Texas MD Anderson Cancer Center, Houston, TX
}

In this issue of Annals of Surgical Oncology, Ronellenfitsch et al. ${ }^{1}$ report the results of a single-arm, phase II window-of-opportunity (WOO) study of preoperative pazopanib in patients with high-risk soft tissue sarcoma (STS). Treatment-naïve patients with localized, resectable, intermediate- or high-grade STS at least $5 \mathrm{~cm}$ in size were eligible to receive neoadjuvant pazopanib $800 \mathrm{mg}$ for 21 days, followed by surgery 7-14 days after the last dose. The primary endpoint of the study was to evaluate the metabolic response rate (MRR), defined as a $\geq 50 \%$ reduction in mean standardized uptake value (SUV) of tumor areas between baseline and post-treatment [18F]fluorodeoxyglucose-positron emission tomography/computed tomography (FDG-PET/CT). Twenty-one of the target 35 patients were enrolled prior to study termination based on the results of a planned futility analysis. Of 17 evaluable patients, one patient achieved $\mathrm{a} \geq 50 \%$ reduction in mean SUV [MRR 5.9\%, 95\% confidence interval (CI) $<0.01-0.29]$. It is also important to note that preoperative pazopanib did not appear to negatively impact tumor resectability, quality of resection, or perioperative outcomes. Most patients enrolled (19/21) underwent planned resection, all with negative margins, and with an acceptable profile of treatment-related toxicities (7/21 patients, $33.3 \%$ with grade 3 or 4 ) and postoperative complications (1 patient, grade 4).

As this study highlights, WOO studies provide unique opportunities for evaluating novel treatments in untreated patients, particularly where large randomized trials are not feasible, such as in rare malignancies. In a WOO design,

(C) Society of Surgical Oncology 2019

First Received: 15 January 2019;

Published Online: 24 January 2019

C. L. Roland, MD, MS

e-mail: clroland@mdanderson.org treatment-naïve patients are exposed to novel agents for a short period of time ( $<1$ month) prior to definitive treatment (often surgery), at which time biologic activity is ascertained. Unlike traditional neoadjuvant studies where the endpoint of the trial is disease outcome, WOO studies utilize biologic primary endpoints ascertained at the time of surgery. The endpoint can be radiographic, such as in the current study by Ronellenfitsch et al. or pathologic, such as change in $\mathrm{Ki}-67,{ }^{2}$ pathologic complete response ${ }^{3}$ or hyalinization. ${ }^{4}$ Importantly, a primary endpoint with a statistical hypothesis is mandatory, similar to other clinical trial designs. Safety endpoints with early stopping rules should be predefined and should include surgical outcomes.

We applaud the authors for undertaking this study to evaluate preoperative pazopanib in treatment-naïve, resectable STS, particularly in view of the widely recognized challenges of designing and executing exploratory studies in STS. The disappointing response rate to pazopanib observed in this study compared with that achieved in other landmark studies, leading to the approval of this anti-angiogenic tyrosine kinase inhibitor, ${ }^{5-7}$ is intriguing. Indeed, as the authors note, there may be as yet poorly understood biologic differences between earlier stage, localized STS compared with metastatic STS, leading to differential response to therapy. Additionally, the time point chosen for metabolic response assessment may have been too early to detect treatment response. Finally, the STS histologies represented in this study not only differ from those of prior studies of pazopanib in distribution, as the authors point out, but are also highly heterogeneous [11 dedifferentiated liposarcomas (LPSs), 2 pleomorphic LPSs, 1 myxoid LPS, 3 undifferentiated pleomorphic sarcomas, 1 leiomyosarcoma, 1 malignant peripheral nerve sheath tumor, 1 synovial sarcoma, 1 fibrohistiocytic sarcoma] in a study already limited by small numbers. Thus, although this was a negative study, we feel it is important to note 
that the majority of the 17 evaluable patients exhibited some decrease in mean SUV, which may be worth further investigation in larger, more homogeneous STS cohorts.

It is well-established that STS encompasses a highly heterogeneous group of malignancies with $>50$ histologic subtypes characterized by unique biologic differences, clinical behaviors, therapeutic vulnerabilities, and prognoses. The discussion above regarding the challenges in this study should not be taken as critical, but rather are meant to highlight the importance of thoughtful clinical trial design in rare malignancies where significant heterogeneity and disease biology must be balanced with meeting accrual targets. Unanswered questions include the following. What is the best measure of response? How does radiographic response correlate with pathologic response? Is metabolic response different among different histologies? What is the optimal timing of treatment prior to surgery?

As we are entering the era of personalized medicine, neoadjuvant therapy and WOO trials provide the opportunity to assess individual patient response to therapy and help tailor adjuvant treatment recommendations. This approach to systemic therapy also provides the tremendous opportunity to collect longitudinal blood and tumor specimens, including the bulk tumor specimen at the time of definitive resection, across the duration of treatment, and facilitate biomarker identification and assessment and allow investigators to study mechanisms of response and resistance to treatment. ${ }^{8}$ Importantly, WOO studies enroll patients who are treatment-naïve or less heavily pretreated, and are opportunities to interrogate tumor biology and response to treatment at an earlier stage of disease before selective pressures and secondary tumor evolution has occurred.

\section{REFERENCES}

1. Ronellenfitsch U, Karampinis I, Dimitrakopoulous-strauss A, Sachpekidis C, Jakob J, Kasper B, et al. Preoperative pazopanib in high-risk soft tissue sarcoma (STS): phase II window-ofopportunity study of the German Interdisciplinary Sarcoma Group (NOPASS/GISG0-4). Ann Surg Oncol. 2019.

2. Schmid P, Pinder SE, Wheatley D, Macaskill J, Zammit C, Hu J, et al. Phase II Randomized Preoperative Window-of-Opportunity Study of the PI3 K Inhibitor Pictilisib Plus Anastrozole Compared With Anastrozole Alone in Patients With Estrogen ReceptorPositive Breast Cancer. J Clin Oncol. 2016;34(17):1987-94.

3. Amaria RN, Reddy SM, Tawbi HA, Davies MA, Ross MI, Glitza IC, et al. Neoadjuvant immune checkpoint blockade in high-risk resectable melanoma. Nat Med. 2018;24(11):1649-1654.

4. Keung EZ, Lazar AJ, Torres KE, Wang W-L, Cormier JN, Ashleigh Guadagnolo B, et al. Phase II study of neoadjuvant checkpoint blockade in patients with surgically resectable undifferentiated pleomorphic sarcoma and dedifferentiated liposarcoma. BMC Cancer. 2018;18(1):913.

5. van der Graaf WT, Blay J-Y, Chawla SP, Kim D-W, Bui-Nguyen B, Casali PG, et al. Pazopanib for metastatic soft-tissue sarcoma (PALETTE): a randomised, double-blind, placebo-controlled phase 3 trial. Lancet. 2012;379(9829):1879-86.

6. Sleijfer S, Ray-Coquard I, Papai Z, Le Cesne A, Scurr M, Schöffski P, et al. Pazopanib, a multikinase angiogenesis inhibitor, in patients with relapsed or refractory advanced soft tissue sarcoma: a phase II study from the European organisation for research and treatment of cancer-soft tissue and bone sarcoma group (EORTC study 620). J Clin Oncol. 2009;27(19):3126-32.

7. Samuels BL, Chawla SP, Somaiah N, Staddon AP, Skubitz KM, Milhem MM, et al. Results of a prospective phase 2 study of pazopanib in patients with advanced intermediate-grade or highgrade liposarcoma. Cancer. 2017;123(23):4640-7.

8. Shiozawa K, Yoshioka Y, Qiao Z, Shuting J, Ochiya T, Kondo T. Pazopanib-induced changes in protein expression signatures of extracellular vesicles in synovial sarcoma. Biochem Biophys Res Commun. 2018;506(3):723-30.

Publisher's Note Springer Nature remains neutral with regard to jurisdictional claims in published maps and institutional affiliations. 